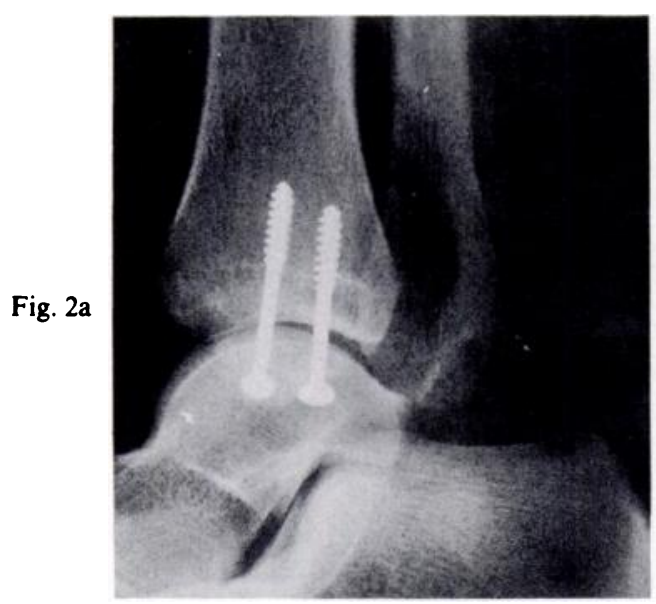

ligament). Perry et al also stated that if the dislocated fibula did not fracture, the medial structures would remain intact. They referred, as support for their theory, to the three cases with an intact dislocated fibula reported in the literature, none of which had damage to the medial structures, and suggested that these three cases represented an earlier stage in the development of the complete injury. Our case, however, in which posterior dislocation of an intact fibula was combined with a medial malleolar fracture seems to contradict the proposed mechanism.

It is unlikely that external rotation of a supinated foot could cause posterior dislocation of the fibula and a medial malleolar fracture. Such an injury might result from external rotation of a pronated foot, or the position of the foot might have changed during the injury, from supination to pronation. Perhaps local anatomical variations also play a role.

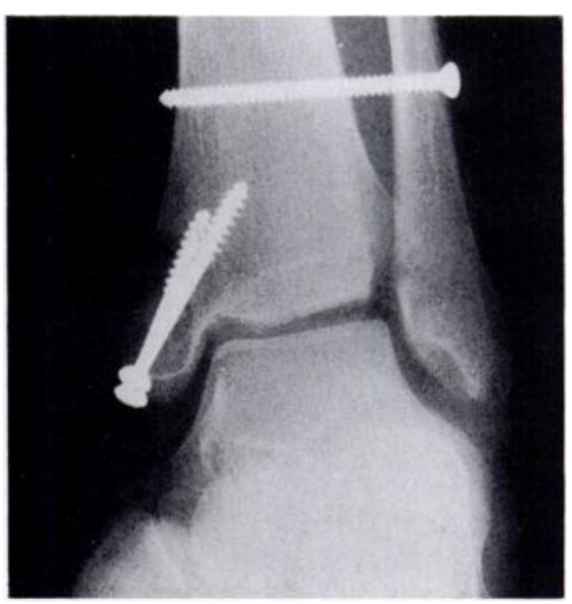

Fig. $2 b$
Since the exact nature of this type of injury still remains unclear and because of the severity of the associated ligamentous damage, there is no place for nonoperative management. We believe that it is wise to explore both sides of the ankle and repair the damaged structures both medially and laterally.

No benefits in any form have been received or will be received from a commercial party related directly or indirectly to the subject of this article.

\section{REFERENCES}

Bosworth DM. Fracture-dislocation of the ankle with fixed displacement of the fibula behind the tibia. J Bone Joint Surg 1947; 29:130-5.

Perry CR, Rice S, Rao A, Burdge R. Posterior fracture-dislocation of the distal part of the fibula: mechanism and staging of injury. $J$ Bone Joint Surg [ Am] 1983; 65-A :1149-57.

Schatzker J, McBroom R, Dzioba R. Irreducible fracture dislocation of the ankle due to posterior dislocation of the fibula. J Trauma 1977; $17: 397-401$.

\title{
THE KNEE FLEXION TEST: A NEW TEST FOR LUMBOSACRAL ROOT TENSION
}

\section{F. POSTACCHINI, G. CINOTTI, S. GUMINA}

The usual clinical tests to detect tension and irritation in the nerve roots forming the femoral or the sciatic nerve are well known. The straight-leg-raising (SLR) test and the femoral-nerve-stretch test involve the L5, S1 or S2 roots and the $\mathrm{L} 2$ to $\mathrm{L} 4$ roots respectively.

We report a new test, the knee-flexion test, which

F. Postacchini, Professor of Orthopaedic Surgery

Orthopaedic Clinic, University of Modena, Largo del Pozzo 71, 41100

Modena, Italy.

G. Cinotti, Registrar

S. Gumina, Resident

I Orthopaedic Clinic, University of Rome, 'La Sapienza', Piazzale Aldo Moro 5, 00185 Rome, Italy.

Correspondence to Professor F. Postacchini.

(C)1993 British Editorial Society of Bone and Joint Surgery

0301-620X/93/5R79\$2.00

J Bone Joint Surg [Br] 1993; 75-B:834-5.

Received 9 December 1992; Accepted 13 January 1993 indicates tension in the lumbosacral roots. It is positive when, with the patient in the prone position, flexion of the knee causes or aggravates pain in the ipsilateral buttock and/or the posterior aspect of the thigh (Fig. 1). A positive test appears to be a reliable predictor of herniation of a lower lumbar disc.

Patients, methods and results. Over a three-year period we examined 512 patients with clinical evidence of lumbar disc herniation. Tension tests, including the SLR and the knee-flexion tests were performed in each patient, and the results were graded as negative, slight, moderate or marked.

The knee-flexion test was positive in 48 patients (29 men and 19 women aged from 22 to 57 years). Of these, 34 underwent CT, MRI and/or myelography; seven refused these investigations and seven did not return for further evaluation. In 32 of the 34 patients, imaging 


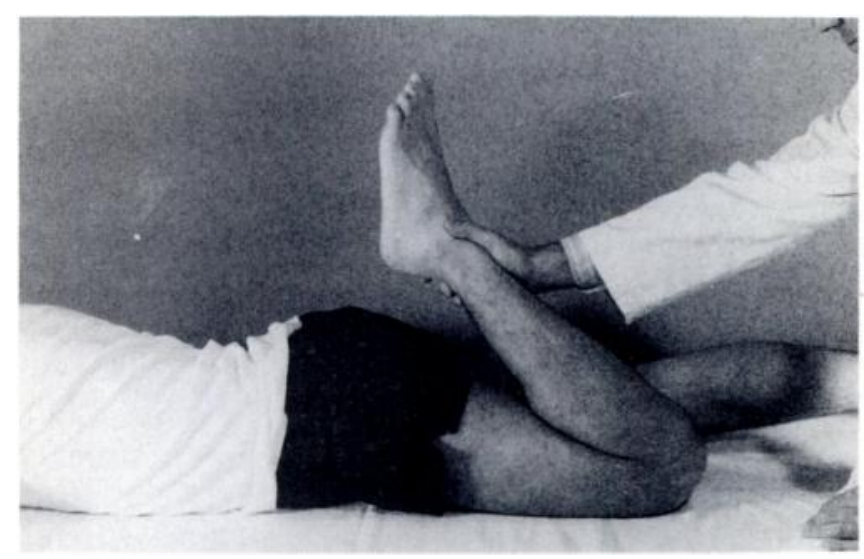

Fig. 1

studies showed disc herniation at L4-L5 or L5-S1. Of the other two patients, both of whom had a slightly positive knee-flexion test, one had a mild disc bulge at L4-L5 and the other had no evidence of disc disease. Of the 32 patients with positive studies, 27 had a large protrusion or an extruded or sequestrated fragment; the other five had medium or small disc protrusions. A moderately or markedly positive knee-flexion test correlated well with an extruded or sequestrated disc. The SLR test was positive in 28 of the 32 patients and negative or equivocal in four. No patient had simultaneous positive results from the femoral-nerve-stretch test and the knee-flexion test.

In the 464 patients with a negative knee-flexion test, neuroradiological studies were available for 246 , and disc herniation at L4-L5 or L5-S1 or both was present in 184 .

The sensitivity, specificity and predictive values of the knee-flexion test for lower lumbar disc herniation were calculated. This showed the test to have a low sensitivity $(55 \%)$ and specificity $(25 \%)$, but a very high predictive value $(94 \%)$. Surgery was required for $84 \%$ of the 32 patients with a positive knee-flexion test compared with $59 \%$ of those with a negative test.

Discussion. The clinical diagnosis of lumbar disc herniation and the decision to perform CT or MRI may be difficult in some patients. The knee-flexion test may be extremely useful in this regard. When the knee-flexion test is positive, there is likely to be a lower lumbar disc herniation which will probably need operative treatment. Almost all our patients with a positive test had a herniated disc, and most needed surgery.

The mechanism of production of sciatic pain by this test is unknown. It may be that knee flexion in the prone position stretches not only the high lumbar roots, but also, to a minimal extent, the lumbosacral roots; slight movements in the presence of severe compression could elicit sciatic pain. It is also possible that stretching of the lumbar plexus pulls on the sacral plexus through the interconnecting branches. This may occur when the anastomoses are larger or more numerous than normal, or the L5 root is included in the lumbar plexus (Testut 1949). If this is so it may explain why the test is positive in only a minority of patients with lower lumbar disc herniation.

No benefits in any form have been received or will be received from a commercial party related directly or indirectly to the subject of this article.

\section{REFERENCE}

Testut JL. In: Latarjet A, Latarjet M, eds. Traitè d'anatomie humaine. Seventh ed. Paris: Doin et Cie, 1949.

\title{
FAILURE OF THE MECRON SCREW-IN RING
}

\author{
W. N. CAPEllo, R. A. COLyeR, C. B. KERNEK, J. V. CARNAHAN, J. J. HESS
}

High failure rates of cemented acetabular components in patients followed for more than ten years, and of cemented acetabular components used for their revision prompted the search for an alternative means of securing

\footnotetext{
W. N. Capello, MD, Professor

R. A. Colyer, MD, Associate Professor

C. B. Kernek, MD, Associate Professor

J. J. Hess, BA, Clinical Research Coordinator

Department of Orthopaedic Surgery, Indiana University Medical Centre, Clinical Building, Room 600, 541 Clinical Drive, Indianapolis, Indiana 46202-5111, USA.

J. V. Carnahan, $\mathrm{PhD}$,

Department of General Engineering, University of Illinois at UrbanaChampaigne, Urbana, Illinois 61801, USA.

Correspondence to Professor W. N. Capello.
}

(C) 1993 British Editorial Society of Bone and Joint Surgery 0301-620X/93/5R74 \$2.00

J Bone Joint Surg [Br] 1993; 75-B : 835-6.

Received 18 June 1992; Accepted after revision 17 August 1992 the acetabular component to bone. The Mecring (Mecron, Berlin, Germany), a titanium-threaded ring (Fig. 1), was introduced in the United States in 1982 in the hope that it would provide firm, immediate fixation without undue sacrifice of bone stock.

We report our experience with this device especially in young patients and those undergoing revision surgery. Patients and methods. Between August 1984 and October 1985,86 Mecron rings were implanted in 79 patients (43 men; 36 women). Their average age at the time of surgery was 54 years (25 to 84). Fifty-four hips underwent primary replacement and 32 had revisions. The femoral component was cemented in 12 and uncemented in 74 hips. The average follow-up of the hips not revised was $\mathbf{4 2}$ months (26 to 51 ).

The posterolateral approach was used in 58 hips; the direct lateral approach (Hardinge 1982) in 28. The implantation technique was that recommended by the 